This is the accepted manuscript, which has been accepted by IEEE for publication (C) 2014. Personal use of this material is permitted. Permission from IEEE must be obtained for all other uses, in any current or future media, including reprinting/republishing this material for advertising or promotional purposes, creating new collective works, for resale or redistribution to servers or lists, or reuse of any copyrighted component of this work in other works. The full reference is:

\title{
"Probabilistic Small-disturbance Stability Assessment of Uncertain Power Systems Using Efficient Estimation Methods"
}

\author{
R. Preece, K. Huang, J.V. Milanović
}

IEEE Transactions on Power Systems, vol. 29, issue 5, pp. 2509-2517, 2014

Digital Object Identifier: 10.1109/TPWRS.2014.2308577 


\title{
Probabilistic Small-disturbance Stability Assessment of Uncertain Power Systems Using Efficient Estimation Methods
}

\author{
Robin Preece, Member, IEEE, Kaijia Huang, and Jovica V. Milanović, Fellow, IEEE
}

\begin{abstract}
The paper presents comparative analysis of performance of three efficient estimation methods when applied to the probabilistic assessment of small-disturbance stability of uncertain power systems. The presence of uncertainty in system operating conditions and parameters results in variations in the damping of critical modes and makes probabilistic assessment of system stability necessary. The conventional Monte Carlo (MC) approach, typically applied in such cases, becomes very computationally demanding for very large power systems with numerous uncertain parameters. Three different efficient estimation techniques are therefore compared in this paper - point estimation methods (PEM), an analytical cumulant-based approach, and the probabilistic collocation method (PCM) - to assess their feasibility for use with probabilistic small disturbance stability analysis of large uncertain power systems. All techniques are compared with each other and with a traditional numerical MC approach and their performance illustrated on a multi-area meshed power system.
\end{abstract}

Index Terms - Cumulant, eigenvalues, electromechanical oscillations, Monte Carlo, point estimation, probabilistic collocation method, small disturbance stability, uncertainty.

\section{LIST OF SYMBOLS}

C set of coefficients used in the PCM.

F conditional probability density function.

$\boldsymbol{H}$ matrix of orthogonal polynomials used in the PCM.

$H_{n}(\bullet) \quad n^{\text {th }}$ order orthogonal polynomial of a function.

$m \quad$ number of uncertain parameters.

$o \quad$ order of PCM model implemented.

$w \quad$ weight value associated with the PEM.

$Y \quad$ uncertain distributed system output parameter.

$\hat{Y} \quad$ estimate of $Y$.

$\alpha \quad$ raw moment of a randomly distributed variable.

$\beta \quad$ central moment of randomly distributed variable.

$\Gamma \quad$ set of uncertain system input parameters.

$\gamma \quad$ uncertain system input parameter.

$\delta_{\text {crit }} \quad$ damping of the critical electromechanical mode.

$\varepsilon$ ARMS average root mean squared error

This work was supported by the Research Councils UK, through the HubNet consortium (grant number: EP/I013636/1).

R. Preece, Kaijia Huang, and J.V. Milanović are with the School of Electrical and Electronic Engineering, The University of Manchester, PO Box 88, Manchester, M60 1QD, UK. (email: robin.preece@manchester.ac.uk, milanovic@manchester.ac.uk).

\author{
$\eta \quad$ normal distribution with $\mu=0$, and $\sigma=1$. \\ $\kappa \quad$ cumulant of a randomly distributed variable. \\ $\mu \quad$ mean of a randomly distributed variable. \\ $\xi \quad$ standard locations associated with the PEM. \\ $\sigma \quad$ standard deviation of a randomly distributed variable. \\ $\psi \quad$ standardized moment of randomly distributed variable.
}

\section{INTRODUCTION}

$\mathrm{P}$ OWER systems are operated in increasingly uncertain conditions as stochastic and intermittent renewable energy sources see greater levels of penetration and new load types are introduced. The effect of these uncertain conditions and system parameters on all aspects of system performance needs be thoroughly investigated and quantified. Probabilistic studies provide a way in which to include these uncertainties in system planning and operation analysis and yield a better depiction of expected system variation than conventional deterministic approaches.

Low frequency oscillations are inherent to large power systems [1] and they can often be exacerbated by the use of fast acting, high gain, generator exciters introduced to improve power system transient recovery [2]. As complex conditions evolve within uncertain power systems, these underlying oscillatory modes can become poorly damped or even unstable. This can lead to equipment disconnection, loss of supply and, in some cases, eventual system collapse.

Due to increased uncertainties associated with the operation of modern power systems, probabilistic approaches towards small-disturbance stability analysis have started to receive greater research attention recently, e.g. [3]-[5]. The benefits of the probabilistic approach are evident and result in more accurate depictions of the true modal variation. The dependence of the methods proposed in [3], [4] on numerical studies, however, severely limits their potential applications. The need for a large number of numerical simulations (typically thousands) means that the computational burden of the technique can often be too high for repeated probabilistic studies, or online applications. A more efficient approach was proposed first in [6] and implemented more recently in [5]. It utilizes tetrachoric series in order to generate a probabilistic stability region for a power system. This work, however, is limited in its assumption that all electromechanical modes can be described by a multivariate normal distribution which does not generally hold for non-linear power systems. 
Efficient estimation techniques which do not impose parametric output distributions include point estimate methods (PEM) [7]-[10], analytical cumulant-based approaches [11][14], and the probabilistic collocation method (PCM) [15][17]. These methods have typically been applied within the area of probabilistic load flow, though some have also been used to analyse the effects of uncertainties on smalldisturbance studies studies, such as [10]-[12] and [17]. All these techniques can be used to derive the probability density functions ( $p d f s)$ of an uncertain system output, based on known input uncertainty, using signifcantly fewer sample points than are required for traditional numerical Monte Carlo (MC) approaches. This significant reduction in the number of required sample points means that these methods are fast enough for online application and repeated probabilistic studies (though this will be ultimately dependent on system size and complexity).

This paper presents the first comparative analysis of the reported efficient estimation techniques against each other in order to establish their applicability to probabilistic smalldisturbance stability studies. Previous studies such as [10][12] and [17] have compared individual methods against the numerical MC approach, however a comparison of these estimation techniques against each other has not been done. These methods are referred to as efficient throughout this paper to differentiate them from the traditional numerical approach - however it should be noted that the accuracy of these techniques is not guaranteed. The accuracy of the efficient approaches at producing both descriptive moments and entire $p d f s$ of system outputs, for differing levels of system parameter uncertainty, is tested on a multi-area meshed power system. Based on the comprehensive results obtained, recommendations are made regarding suitability of the examined methods for probabilistic small disturbance stability analysis of uncertain power systems.

\section{EFFicient Estimation TeChNiQUes fOR Probabilistic SMALL-DISTURBANCE STABILITY ANALYSIS}

Small-disturbance stability relates to the ability of a power system to maintain synchronous operation when subjected to the small disturbances that occur constantly during operation [18]. By linearizing the power system model and calculating the eigenvalues of the state matrix, the system modes of oscillation can be identified. In large power systems, low frequency, inter-area, electromechanical oscillations are typically the least damped most persistent modes which dominate post-disturbance system behavior and therefore, represent the critical modes in the system. Conventional, deterministic, small-disturbance stability analysis identifies and characterizes these modes. Probabilistic small-disturbance stability analysis incorporates system uncertainties (e.g., in system loading or power generation from renewable energy sources) in small-disturbance stability assessment in order to produce statistical distributions of critical modes which more accurately describe the behavior of uncertain power systems.

There are a variety of efficient estimation techniques that can be used for probabilistic small-disturbance stability analysis [7]-[17]. This section presents a brief theoretical background on several of the most promising and commonly used methods. These methods are further investigated and their application illustrated in subsequent sections. In all cases it is assumed that the uncertain parameter set $\Gamma$ is known and that probability density functions are fully detailed for each uncertain input parameter $\gamma_{j} \in \Gamma$.

Throughout this section, reference is made to raw moments $\alpha$, central moments $\beta$, and standardized moments $\psi$ of distributions. The following definitions apply for a random variable $x$ with probability density function $f(x)$ [19].

The $n^{\text {th }}$ order raw moment $\alpha_{n}^{x}$ is given by (1). The first raw moment is the mean $\mu_{x}$.

$$
\alpha_{n}^{x}=\int_{-\infty}^{\infty} x^{n} f(x) d x
$$

The $n^{\text {th }}$ order central moment $\beta_{n}^{x}$ is given by (2). The second central moment is the variance $\sigma_{x}^{2}$.

$$
\beta_{n}^{x}=\int_{-\infty}^{\infty}\left(x-\mu_{x}\right)^{n} f(x) d x
$$

The $n^{\text {th }}$ order standardized moment $\psi_{n}^{x}$ is given by (3). The third and fourth standardized moments are the measures of skewness and kurtosis of the distribution, respectively.

$$
\psi_{n}^{x}=\frac{\beta_{n}^{x}}{\sigma_{x}{ }^{n}}
$$

\section{A. Monte Carlo (Numerical) Method}

The numerical Monte Carlo (MC) approach is used as the benchmark against which the other methods described in this paper are assessed (in terms of both accuracy and computational burden). This approach relies on extensive and repeated random sampling of system uncertainties in order to obtain a large data set from which output $p d f s$ can be determined [20].

For each input set, randomly generated using the MC approach, a deterministic study is performed (consisting of load flow, system linearization, eigenvalue analysis, and modal identification) in order to calculate the details of critical system modes. As the number of samples increases, it becomes increasingly probable that the distribution of output variation is an accurate representation of the true variation. It is therefore necessary to run large numbers of full deterministic studies, which can limit the application of the MC method when performing probabilistic studies of large uncertain power systems. Efficient sampling techniques aim to reduce the number of deterministic studies required, whilst maintaining the accuracy of the results produced.

\section{B. Point Estimate Method}

A number of Point Estimation Methods (PEM) have been developed, with varying levels of application to probabilistic power system research [7]-[9]. The aim of all point estimate techniques is to compute the moments of the system output $Y$ that is a function of $m$ uncertain input variables. The distribution of $Y$ can be subsequently established using a variety of expansion techniques. 
The PEM techniques used within this study are taken from [21] and were shown to be effective for probabilistic load flow studies in [9]. These PEM approaches can be used with symmetric or asymmetric variables but not with correlated system uncertainties. Other PEM approaches also exist and if input correlation is essential, different PEM techniques such as [22]-[24] may be more suitable - however accuracy and efficiency may be sacrificed. PEM variants are developed in [21] where $K m$ or $K m+1$ full deterministic studies are required (where $K$ is a positive integer). The variants that can be used are dependent on the distribution of input uncertainties. In this study (considering input uncertainties with Gaussian distributions), both the $2 m$ and $4 m+1$ PEM variants are investigated. The PEM details are briefly presented below, with full details given in [9] and [21].

\section{1) Theoretical Background}

Deterministic studies are required at $K m$ or $K m+1$ separate operating points (termed concentrations). The $k^{\text {th }}$ concentration $\left(\gamma_{j, k}, w_{j, k}\right)$ is defined as a pair consisting of a location $\gamma_{j, k}$ and an associated weight value $w_{j, k}$. This location represents the $k^{\text {th }}$ value that the variable $\gamma_{j}$ will take during full deterministic studies.

For PEM deterministic studies, only one input variable is varied at a time and the remaining uncertainties in $\Gamma$ take their mean values $\mu_{\gamma_{j}}$. Therefore only $K m$ deterministic studies are required, i.e., $K$ variations of each of the $m$ uncertain inputs. In the $K m+1$ PEM variants, an additional deterministic study is completed with all uncertainties at their mean values.

Locations are determined using (4), in which $\mu_{\gamma_{j}}$ and $\sigma_{\gamma_{j}}$ are the mean and standard deviation (st.d.) of the $j^{\text {th }}$ uncertain parameter $\gamma_{j}$, and $\xi_{j, k}$ is the standard location.

$$
\gamma_{j, k}=\mu_{\gamma_{j}}+\xi_{j, k} \sigma_{\gamma_{j}}
$$

Standard locations and weights are determined by solving the nonlinear equations (5) [21].

$$
\left.\begin{array}{c}
\sum_{k=1}^{K} w_{j, k}=\frac{1}{m} \\
\sum_{k=1}^{K} w_{j, k}\left(\xi_{j, k}\right)^{n}=\psi_{j, j}^{\gamma} \quad n=1, \ldots,(2 K-1)
\end{array}\right\}
$$

In (5), $\psi_{j, n}^{\gamma}$ is the $n^{\text {th }}$ standardized moment of the $j^{\text {th }}$ uncertain parameter $\gamma_{j}[21]$. As per standard definitions, $\psi_{j, 1}^{\gamma}=0$, $\psi_{j, 2}^{\gamma}=1, \quad \psi_{j, 3}^{\gamma}$ is the skewness, and $\psi_{j, 4}^{\gamma}$ is the kurtosis of $\gamma_{j}$. Equation (5) can only be solved analytically for $K=2$. For $K>2$ numerical solutions are required.

Full deterministic studies are performed for each concentration at the operating point $\left(\mu_{\gamma_{1}}, \mu_{\gamma_{2}}, \ldots, \gamma_{j, k}, \ldots, \mu_{\gamma_{\mathrm{m}}}\right)$ to obtain the value of the system output $Y(j, k)$ for that concentration. These values are subsequently combined with the previously determined weight factors using (6) to determine the $n^{\text {th }}$ raw moment $\alpha_{n}^{Y}$ of the system output $Y$.

$$
\alpha_{n}^{Y}=E\left[Y^{n}\right] \cong \sum_{j=1}^{m} \sum_{k=1}^{K} w_{j, k}(Y(j, k))^{n}
$$

The raw moments $\alpha_{n}^{Y}$ can be used to establish the central moments $\beta_{n}^{Y}$ of the system output. If sufficient moments are estimated, the $p d f$ of $Y$ can be estimated using a suitable expansion. However, the accuracy of PEM approaches typically deteriorates as the order of the estimated raw moment increases [9]. Distributions produced using this approach will, therefore, be more accurate if they are predominantly described by low order moments.

\section{2) $2 m$ Variant}

For the $2 m$ PEM variant, only the first three standardized moments $\psi^{\gamma}$ of each uncertain input are required. The standard locations and weights of the uncertain input $\gamma_{j}$ are calculated using (7) and (8) [9].

$$
\begin{gathered}
\xi_{j, k}=\frac{\psi_{j, 3}^{\gamma}}{2}+(-1)^{k+1} \sqrt{m+\left(\psi_{j, 3}^{\gamma} / 2\right)^{2}} \\
w_{j, 1}=\frac{-\xi_{j, 2}}{m\left(\xi_{j, 1}-\xi_{j, 2}\right)} \quad w_{j, 2}=\frac{\xi_{j, 1}}{m\left(\xi_{j, 1}-\xi_{j, 2}\right)}
\end{gathered}
$$

\section{3) $4 m+1$ Variant}

For the $4 m+1$ PEM variant, the standard locations are calculated as the roots of the polynomial (9), where the coefficients are found by solving the linear equations in (10) [9].

$$
\begin{gathered}
f(\xi)=\xi^{4}+C_{3} \xi^{3}+C_{2} \xi^{2}+C_{1} \xi+C_{0} \\
\left(\begin{array}{cccc}
0 & 1 & \psi_{j, 3}^{\gamma} & \psi_{j, 4}^{\gamma} \\
1 & \psi_{j, 3}^{\gamma} & \psi_{j, 4}^{\gamma} & \psi_{j, 5}^{\gamma} \\
\psi_{j, 3}^{\gamma} & \psi_{j, 4}^{\gamma} & \psi_{j, 5}^{\gamma} & \psi_{j, 6}^{\gamma} \\
\psi_{j, 4}^{\gamma} & \psi_{j, 5}^{\gamma} & \psi_{j, 6}^{\gamma} & \psi_{j, 7}^{\gamma}
\end{array}\right)\left(\begin{array}{l}
C_{0} \\
C_{1} \\
C_{2} \\
C_{3}
\end{array}\right)=-\left(\begin{array}{l}
\psi_{j, 5}^{\gamma} \\
\psi_{j, 6}^{\gamma} \\
\psi_{j, 7}^{\gamma} \\
\psi_{j, 8}^{\gamma}
\end{array}\right)
\end{gathered}
$$

The weights are subsequently determined by solving (11), with the weight value $w_{0}$ in (12) used for the ' +1 ' operating point where all uncertainties take their mean values.

$$
\begin{gathered}
\left(\begin{array}{cccc}
\xi_{j, 1} & \xi_{j, 2} & \xi_{j, 3} & \xi_{j, 4} \\
\xi_{j, 1}^{2} & \xi_{j, 2}^{2} & \xi_{j, 3}^{2} & \xi_{j, 4}^{2} \\
\xi_{j, 1}^{3} & \xi_{j, 2}^{3} & \xi_{j, 3}^{3} & \xi_{j, 4}^{3} \\
\xi_{j, 1}^{4} & \xi_{j, 2}^{4} & \xi_{j, 3}^{4} & \xi_{j, 4}^{4}
\end{array}\right)\left(\begin{array}{l}
w_{j, 1} \\
w_{j, 2} \\
w_{j, 3} \\
w_{j, 4}
\end{array}\right)=\left(\begin{array}{c}
0 \\
1 \\
\psi_{j, 3}^{\gamma} \\
\psi_{j, 4}^{\gamma}
\end{array}\right) \\
w_{0}=1-\sum_{j}^{m} \sum_{k=1}^{K} w_{j, k}
\end{gathered}
$$

\section{4) Summary}

The PEM approach can be summarized as follows: $i$ ) Calculate concentrations (locations and weights) for each uncertain input using (4), (7) and (8) for the $2 \mathrm{~m}$ variant, and (4), (9)-(12) for the $4 m+1$ variant; ii) Perform deterministic studies at each concentration; iii) Calculate the output raw moments using (6); iv) If desired, calculate output central moments or standard moments and generate $p d f s$.

\section{Cumulant Method}

The cumulants $\kappa$ of a probability distribution provide an alternative mathematical description to the moments of the distribution. The use of cumulants allows an analytical solution to be derived for the output variation based on system 
input uncertainty.

Analytically, the moments of the system output $Y$ are described by a mathematical convolution of the moments of the independent input uncertainties $\Gamma$. This is extremely complex to calculate for high order moments when there are multiple uncertainties. However, the cumulants $\kappa^{Y}$ of the system output can be described simply by a sum of the cumulants $\kappa^{\Gamma}$ of the independent input uncertainties [13]. This is much easier to calculate, and the output cumulants can subsequently be used to establish the output moments.

The cumulant-based approach has been used in power system studies to determine probabilistic load flow solutions in [13], [14], and also for probabilistic small disturbance stability studies in [11], [12]. The method is typically used alongside the Gram-Charlier expansion [25] in order to generate output $p d f s$ from the calculated output cumulants and yielded good results [11]-[14]. The mathematical analysis required, however, is complex, and it is often necessary to include numerical approximations within the calculations in order to converge on a solution within an acceptable length of time.

The cumulant-based approach can also be used with correlated input uncertainties [11], [12]. This has not been investigated in this paper as both the PEM and PCM approaches studied here assume independence of uncertainty distributions.

\section{1) Theoretical Background}

The $n^{\text {th }}$ order cumulants $\kappa_{j, n}^{\gamma}$ of the $j^{\text {th }}$ uncertain parameter $\gamma_{j}$ are determined from its mean $\mu_{\gamma_{j}}$ and central moments $\beta_{j, n}^{\gamma}$ using the standard relationships given by (13) [13].

$$
\begin{aligned}
& \boldsymbol{\kappa}_{j, 1}^{\gamma}=\mu_{\gamma_{j}}, \quad \boldsymbol{\kappa}_{j, 2}^{\gamma}=\beta_{j, 2}^{\gamma}, \quad \boldsymbol{\kappa}_{j, 3}^{\gamma}=\beta_{j, 3}^{\gamma}, \\
& \boldsymbol{\kappa}_{j, 4}^{\gamma}=\beta_{j, 4}^{\gamma}-3\left(\beta_{j, 2}^{\gamma}\right)^{2} \ldots \ldots \ldots .
\end{aligned}
$$

The cumulants of the change in system output $\Delta Y$ can then be calculated directly using (14).

$$
\kappa_{n}^{\Delta Y}=\sum_{j=1}^{m}\left(\partial Y / \partial \gamma_{j}\right)^{n} \kappa_{j, n}^{\gamma}
$$

The sensitivity term $\left(\partial Y / \partial \gamma_{j}\right)$ in (14) can be determined either numerically or analytically. For probabilistic smalldisturbance studies, an analytical sensitivity determination requires knowledge of the full linearized state matrix and is detailed in [12]. Many power system simulation software programs do not provide this, and instead the numerical approximation (15) is required.

$$
\frac{\partial Y}{\partial \gamma_{j}} \cong \frac{Y\left(\mu_{\gamma_{j}}+\Delta \gamma_{j}\right)-Y\left(\mu_{\gamma_{j}}\right)}{\Delta \gamma_{j}}
$$

In (15), $\Delta \gamma_{j}$ represents a small change in the uncertain input $\gamma_{j}$. A $1 \%$ positive variation is used for all uncertain inputs in this study. Numerical calculation of this sensitivity will require $m+1$ deterministic studies for $m$ system uncertainties (once for each change, and one more with all mean values for comparison). This represents the only deterministic studies required for this efficient estimation technique. The numerical calculation of sensitivity is used within this work and the cumulant-based approach can therefore be considered as an analytical technique that utilizes a numerical approximation.

Once sufficient output cumulants have been established, the central moments of $\Delta Y$ (equal to the central moments of $Y$ ) can be calculated using the relationships given by (16) [13]. These moments are used to determine the probability distribution of $\Delta Y$ (and subsequently $Y$ ) using suitable expansions (such as the Gram-Charlier) if required.

$$
\begin{aligned}
& \beta_{1}^{Y}=\beta_{1}^{\Delta Y}=0, \quad \beta_{2}^{Y}=\beta_{2}^{\Delta Y}=\kappa_{2}^{\Delta Y}, \quad \beta_{3}^{Y}=\beta_{3}^{\Delta Y}=\kappa_{3}^{\Delta Y}, \\
& \beta_{4}^{Y}=\beta_{4}^{\Delta Y}=\kappa_{4}^{\Delta Y}+3\left(\kappa_{2}^{\Delta Y}\right)^{2} \ldots \ldots \ldots . .
\end{aligned}
$$

\section{2) Summary}

The cumulant based method can be summarized as follows: i) Calculate uncertain input cumulants based on input mean and input central moments using (13); ii) Perform $m+1$ deterministic studies in order to numerically calculate the sensitivity of the output to each uncertainty using (15); iii) Calculate the cumulants of the change in system output directly using (14); iv) Calculate the central moments of the system output using the relationships detailed in (16); v) If desired, calculate output standard moments and generate $p d f s$.

\section{Probabilistic Collocation Method}

With the Probabilistic Collocation Method (PCM), the approximated system output $\hat{Y}$ is modeled directly a as a polynomial function of the uncertain parameter set $\Gamma$, as in (17).

$$
\hat{Y}=g(\Gamma)
$$

The key aspects of the PCM are (a) the selection of the function, and $(b)$ specifying the points at which to run deterministic power system simulations to gather the data from which the PCM model is formed. These details are provided briefly here and are covered in greater depth in [15]-[17].

\section{1) Theoretical Background}

Orthogonal polynomials of increasing order are derived for the known probability distributions of each uncertain parameter $\gamma_{j}$. The model function $g(\Gamma)$ is formed as a sum of products of these polynomials (selected based on the desired order of the final PCM model) which are weighted by a set of coefficients $\boldsymbol{C}$. Orthogonal polynomials can be created for any known probability density function $(p d f)$. Normally distributed parameters are simply represented by the transformation (18).

$$
\gamma_{j}=\mu_{\gamma_{j}}+\sigma_{\gamma_{j}}\left(H_{1}(\eta)\right)
$$

In (18), $\mu_{\gamma_{j}}$ and $\sigma_{\gamma_{j}}$ are as previous described, and $H_{1}(\eta)$ is the first order orthogonal polynomial of the standard normal distribution $\eta$.

The orthogonal polynomials $H_{n}(\eta)$, where $n$ is the polynomial order, are given by the standard Hermite polynomials [26]. Orthogonal polynomials for other distributions can be found using recursive methods, for example with functions available from the orthpol set for Matlab [27].

The number of combinations $c$ of the orthogonal polynomi- 
als (and therefore the number of coefficients to find) increases with both the number of uncertainties $m$ and the PCM model order $o$, according to (19), where ! is the factorial operation.

$$
c=\left(\begin{array}{c}
m+o \\
m
\end{array}\right)=\frac{(m+o) !}{m ! o !}
$$

This number increases rapidly as $m$ and $o$ increase, and for each new coefficient, a deterministic study must be performed. These deterministic simulations must be run in order to provide the points around which to solve the set of coefficients $C$ and fit the model as shown in (20).

$$
\boldsymbol{Y}=\boldsymbol{H C}
$$

In (20), $\boldsymbol{Y}$ is a vector of observed system output values; and $\boldsymbol{H}$ is a matrix with rows formed by the combinations of orthogonal polynomials for each uncertain parameter evaluated at the points resulting in the corresponding system output in $\boldsymbol{Y}$.

The points for deterministic studies (the collocation points) are selected by taking inspiration from the Gaussian quadrature technique of estimating integrals [28]. The roots of the next higher order polynomial are chosen as the points at which to perform deterministic simulations and collocation points with the greatest probability of occurring are selected. By doing this, the accuracy of the PCM model is concentrated in the regions defined by the $p d f s$ as most likely to occur.

The PCM differs from the point estimation methods and the cumulant-based approach as it does not directly estimate the moments of the system output. Instead, The PCM function can be used as a computationally inexpensive substitute for running a full simulation of the power system during a standard MC based uncertainty study. Output moments, if desired, can subsequently be determined from the data set produced.

It is also possible to extract the $p d f s$ directly from the PCM model functions without using an MC approach. However this requires the inversion of the PCM function. This is not trivial for non-monotonic multi-dimensional functions and often the numerical methods would take so long as to negate all the computational benefits associated with using the PCM.

\section{2) Uncertainty Reduction}

The number of full deterministic studies required, given by (19), increases extremely rapidly as the number of system uncertainties $m$ increases. For example, in order to produce a $3^{\text {rd }}$ order PCM model, 56 simulations are required if $m=5$; 286 simulations are required if $m=10$; and 1771 if $m=20$. This exponential increase is different from the other efficient methods examined where the number of deterministic studies increases linearly with the number of uncertainties. As a result, for larger power systems, a reduction in the number of uncertainties considered is required in order to preserve the advantages of using an efficient estimation method.

All uncertain parameters are ranked using (21) which describes the sensitivity of the damping of the critical mode $\delta_{\text {crit }}$ to the uncertain parameter $\gamma_{j}$, derived from [16].

$$
r_{\sigma_{c r i t} \gamma_{j}}=\left|\frac{\partial \delta_{c r i t}}{\partial \gamma_{j}}\right|\left|\frac{\sigma_{\gamma_{j}}}{\mu_{\gamma_{j}}}\right|
$$

The first term (the sensitivity measure) is determined using a $1 \%$ positive increase in $\gamma_{j}$ from its mean value. The second term weights this sensitivity by the variation in that uncertain parameter. Based on this ranking only the most influential parameters are selected for further studies. The effectiveness of this parameter reduction technique is illustrated in [17].

\section{3) Summary}

The PCM can be summarized as follows: $i$ ) Reduce the number of considered uncertainties (if large) based on ranking completed using (21) and illustrated in [17]; ii) Establish orthogonal polynomials to represent considered system uncertainties based on desired model order; iii) Determine collocation points for each considered system uncertainty using the roots of higher order orthogonal polynomials, and order based on the joint probability density associated with the operating point; iv) Complete sufficient number of deterministic studies to calculate all coefficients for the PCM model as in (20), selecting the most probable collocation points first; $v$ ) Use the PCM model function as a computationally inexpensive substitute for full deterministic studies in a standard MC simulation process to generate a large data set for the system output; vi) If desired, calculate output moments or produce a $p d f$ based on the obtained data set.

\section{TEST SYSTEM}

The efficient estimation methods described within this paper are illustrated using the 16 machine, 68 bus reduced order representation of the New England Test System and the New York Power System (NETS\& NYPS) [29], shown in Fig. 1. All methods and deterministic system studies are performed within the MATLAB/Simulink environment making use of MATPOWER [30] functions to perform power flows.

\section{A. System Description}

Generators G1-8 use slow DC excitation (IEEE-DC1A), G9 is equipped with a fast acting static exciter (IEEE-ST1A) and power system stabilizer (PSS), and the remaining generators G10-16 are under manual excitation as in [29]. All generators are represented by full sixth order models. System loads are modeled as constant impedance. Full system details, generator and exciter parameters are given in [29] with PSS settings for G9 taken from [2].

\section{B. System Uncertainties}

System uncertainties are represented for all generator outputs and system loads within the network. There are therefore a total of 50 uncertain parameters within the test system being investigated (15 generators excluding the slack, and 35 loads). Generator outputs and loads are both modeled using Gaussian distributions with nominal values set as mean values of corresponding Gaussian distributions. Different levels of standard deviation are considered in order to assess the accuracy of the various methods for differing levels of system parameter variation. Load power factors are considered to be constant at the nominal values.

The uncertainties considered in this work are assumed to follow Gaussian distributions. It should be noted that these efficient methods can be used with any continuous distribution 
provided that moments, cumulants and orthogonal polynomials can be derived.

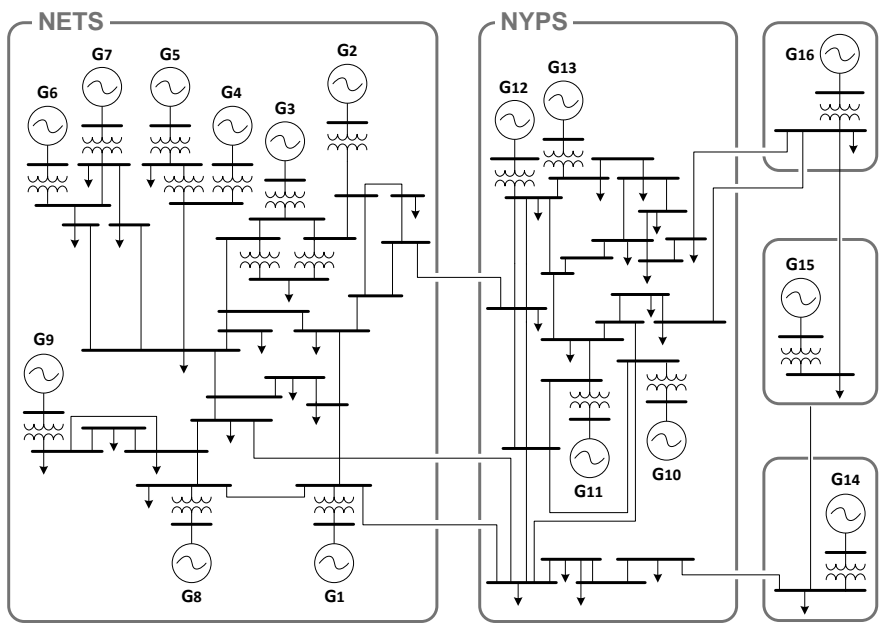

Fig. 1: 16 machine, 68 bus reduced order model of NETS \& NYPS.

\section{Simulation Results}

The test network displays four inter-area modes with damping factors lower than 5\% as detailed in [31]. The analysis presented here focusses on the lowest frequency inter-area mode (of approximately $0.4 \mathrm{~Hz}$ ) which is the critical mode for the system. Specifically, the aim of the simulations is to produce the moments and $p d f s$ for the damping (real part $\delta_{c r i t}$ ) of this critical electromechanical oscillation.

\section{A. Simulation details}

In all cases presented here, comparisons are made relative to the Monte Carlo (MC) numerical approach that was carried out using 10,000 simulations.

Point Estimate Method - The $2 m$ and $4 m+1$ point estimate variants are implemented requiring a total of 100 and 201 deterministic studies respectively.

Cumulant Method - The cumulant-based approach requires a total of 51 deterministic studies to complete the integral sensitivity analysis. The analytical calculation of the critical damping moments was performed using uncertain input cumulants up to $4^{\text {th }}$ order (though higher order cumulants can also be included).

Probabilistic Collocation Method - For the PCM approach, different orders of model function are considered. To enable a balanced comparison, the same number of uncertainties is used for each model. This number is limited by the highest order model considered, which is of $3^{\text {rd }}$ order in this work. The limit imposed in this research is such that the PCM approach does not greatly exceed the number of deterministic studies required by the $4 m+1$ point estimate variant. This restricts the number of considered uncertainties to nine for all models. This results in 10 deterministic studies for the $1^{\text {st }}$ order model, 55 for the $2^{\text {nd }}$ order model, and 220 for the $3^{\text {rd }}$ order model.

The ranking of parameters using the sensitivity-based rank of (21) must first be completed (requiring 51 deterministic studies). The highest ranked uncertainties are selected, creating a reduced set of uncertainties consisting of six generators and three loads. The final PCM models are used as a computationally inexpensive substitute for full deterministic studies in a standard MC approach consisting of 10,000 runs in order to produce the final data sets.

\section{B. Accuracy of Moment Estimation}

The results from each efficient estimation method for the calculated selected moments (mean, st.d., skewness, and kurtosis) are shown in Table 1. These results are shown for a level of input uncertainty variation equal to $5 \%$ at $3 \sigma$ (i.e. $99.7 \%$ of input variation is found within $\pm 5 \%$ of nominal mean values).

A number of features can be observed from the results collated in Table 1. All methods are very accurate at estimating the mean value of $\delta_{\text {crit }}$ with errors never exceeding $0.38 \%$. Estimation of the st.d. is more variable, with errors between $0.66 \%$ (with the cumulant method) to approximately $40 \%$ (with the PCM and $2 m$ PEM variant). It can also be observed that the methods are less accurate at determining higher order moments. The skewness is never estimated more accurately than to within an $87 \%$ error typically cause be estimations near zero. Although the PCM models and cumulant approach can estimate the kurtosis to within $14.8 \%$, the point estimate methods provide results that are over $76 \%$ in error.

TABLE 1

MOMENTS OF MOde DAMPING AND PERCENTAGE ERRORS USING VARIOUS EFFICIENT ESTIMATION METHODS FOR 5\% VARIATION OF UNCERTAINTIES

\begin{tabular}{|c|c|c|c|c|c|}
\hline \multirow{2}{*}{\multicolumn{2}{|c|}{ Method used }} & \multicolumn{4}{|c|}{ Critical Mode Damping Moments } \\
\hline & & $\begin{array}{c}\operatorname{Mean}\left(\mathrm{s}^{-1}\right) \\
\mu_{\delta_{\text {crit }}}\end{array}$ & $\begin{array}{c}\text { St.d. }\left(\mathrm{s}^{-1}\right) \\
\sigma_{\delta_{\text {crit }}}\end{array}$ & $\begin{array}{c}\text { Skewness } \\
\psi_{3}^{\delta_{\text {crit }}} \\
\end{array}$ & $\begin{array}{c}\text { Kurtosis } \\
\psi_{4}^{\delta_{c i i t}} \\
\end{array}$ \\
\hline \multicolumn{2}{|c|}{ Monte Carlo } & -0.1232 & 0.0050 & -0.3274 & 3.3725 \\
\hline \multirow{2}{*}{ PEM } & $2 m$ & -0.1234 & 0.0068 & -4.8405 & 38.3624 \\
\hline & $4 m+1$ & -0.1232 & 0.0049 & -0.0500 & 0.8834 \\
\hline \multicolumn{2}{|c|}{ Cumulant } & -0.1229 & 0.0050 & 0.0028 & 2.9938 \\
\hline \multirow{3}{*}{ PCM } & $1^{\text {st }}$ Order & -0.1229 & 0.0030 & 0.0012 & 3.1146 \\
\hline & $2^{\text {nd }} \mathrm{Order}$ & -0.1229 & 0.0030 & 0.0255 & 3.0283 \\
\hline & $3^{\text {rd }}$ Order & -0.1229 & 0.0030 & 0.0033 & 3.0214 \\
\hline
\end{tabular}

1) Effect of Level of Uncertainty

These moments have also been collated for different levels of input variation with $3 \sigma$ levels of $2.5 \%$ and $7.5 \%$ also considered in addition to the $5 \%$ variation previously shown in Table 1 . The percentage errors of the mean of $\delta_{\text {crit }}$ is shown in Fig. 2 for the different estimation methods at differing levels of variation in input uncertainty.

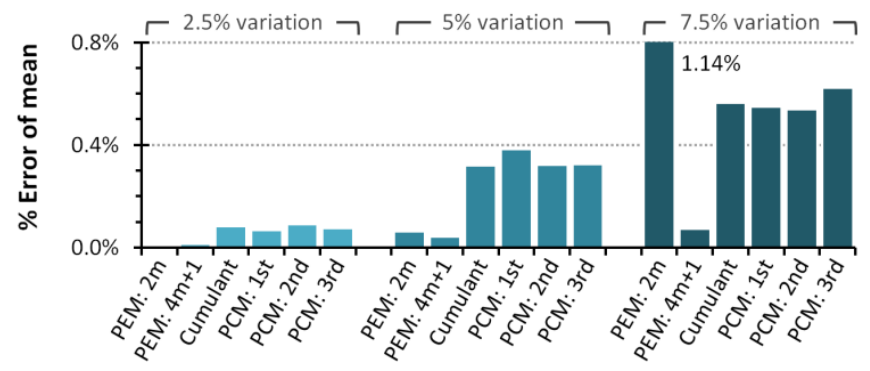

Fig. 2: Error in estimation of mean for different levels of uncertainty.

It can be seen that the error increases with input variability for all methods. It is also evident that the point estimate 
methods display lower error than the cumulant and PCM approaches (with the exception of the $2 m$ PEM variant which becomes the least accurate once input variation reaches $7.5 \%$ ). Very little change is evident between the different order PCM models, suggesting the additional computational requirements of higher order models may not be necessary.

Similar plots could also be produced for the st.d., skewness and kurtosis. With the st.d. estimation, the increase in input variation has the greatest effect on the $2 m$ PEM variant (with an error of $176 \%$ once this input variation reaches $7.5 \%$ ). Errors for the PCM models are relatively unchanged by increasing input uncertainty and are approximately $40 \%$ in all cases with the PCM consistently underestimating the true st.d. of $\delta_{\text {crit. }}$. The $4 m+1$ PEM variant and the cumulant-based approach display the best results. Errors remain below $5.5 \%$ in all cases, even with $7.5 \%$ input variation. It is these two approaches which appear to be the most promising for the efficient estimation of modal parameters.

It should be noted that skewness is poorly estimated by all methods with errors never smaller than $84.5 \%$. Kurtosis is also poorly estimated by both PEM variants with errors greater than $78.0 \%$. However, this is more accurately estimated by the cumulant and PCM approaches with errors below $4.9 \%$ for $2.5 \%$ input variation, below $14.8 \%$ for $5 \%$ input variation, and below $45.3 \%$ when input variation is $7.5 \%$.

\section{Accuracy of Probability Density Function Estimation}

In addition to assessing the accuracy of the estimated moments of $\delta_{\text {crit }}$, the accuracy of the final probability density functions is also assessed. Both the numerical MC approach and the PCM result in the production of large data sets. Probability distributions are subsequently produced using a kernel smoothing density estimate [32].

\section{1) Expansion for PEM Variants}

Due to the large inaccuracy in the estimation of moments above $2^{\text {nd }}$ order using the PEM, only the mean and st.d. are used to generate $p d f s$ for $\delta_{c r i t}$. A Gaussian distribution is, therefore, used for both considered PEM variants. It should be noted that other expansions (such as the Cornish-Fisher [33]) may also provide good results and should be considered when applying these methods to further power system studies. The Cornish-Fisher expansion, though originally considered for this study, was not used due to problem specific parameters which violate restrictions specified in [33] for application of this expansion.

\section{2) Expansion for the Cumulant Approach}

As in previous research [11]-[14], the cumulant approach is combined with the Gram Charlier expansion in order to produce $p d f s$ for $\delta_{c r i t}$. Investigations into expansions of varying order were completed and it was found that no significant improvement in accuracy was gained by using expansions above $4^{\text {th }}$ order - as in [13]. Fourth order Gram-Charlier expansions have therefore been used to produce the cumulantbased $p d f s$ for comparison.

3) Comparison of Probability Density Functions

The $p d f s$ and cumulative probability density functions $(c d f s)$ of $\delta_{c r i t}$ produced using different techniques are shown in
Fig. 3 for the case when uncertain input variation is equal to $5 \%$. Only one plot is shown for the PCM approach as all models produce very similar traces. As predicted from the analysis of the moments, the $4 m+1$ PEM variant and the cumulant-based approach both accurately track the MC-based results. The effect of the underestimation of the st.d. by the PCM and of the overestimation of the st.d. by the $2 m$ PEM variant can clearly be seen - resulting in a loss of accuracy.

To numerically calculate the accuracy of these estimated distributions, the Average Root Mean Square error $\left(\varepsilon_{A R M S}\right)$ measure given by (22) is used.

$$
\varepsilon_{\text {ARMS }}=\sqrt{\frac{\sum_{i=1}^{N}\left(F_{i}^{M C}-F_{i}^{E s t}\right)^{2}}{N}}
$$

In (22), $F_{i}^{M C}$ is the $i^{\text {th }}$ value of the $c d f$ obtained using the Monte Carlo approach, $F_{i}^{E s t}$ is the $i^{\text {th }}$ value of the $c d f$ obtained using an efficient estimation technique, and $N$ is the number of samples considered when calculating $\varepsilon_{A R M S}$. In this study, $\varepsilon_{A R M S}$ is calculated in the range between the $1^{\text {st }}$ and $99^{\text {th }}$ percentiles of $F_{i}^{M C}$ (to avoid large low error tail regions from skewing $\varepsilon_{A R M S}$ towards misrepresentative low values) using 1000 samples.
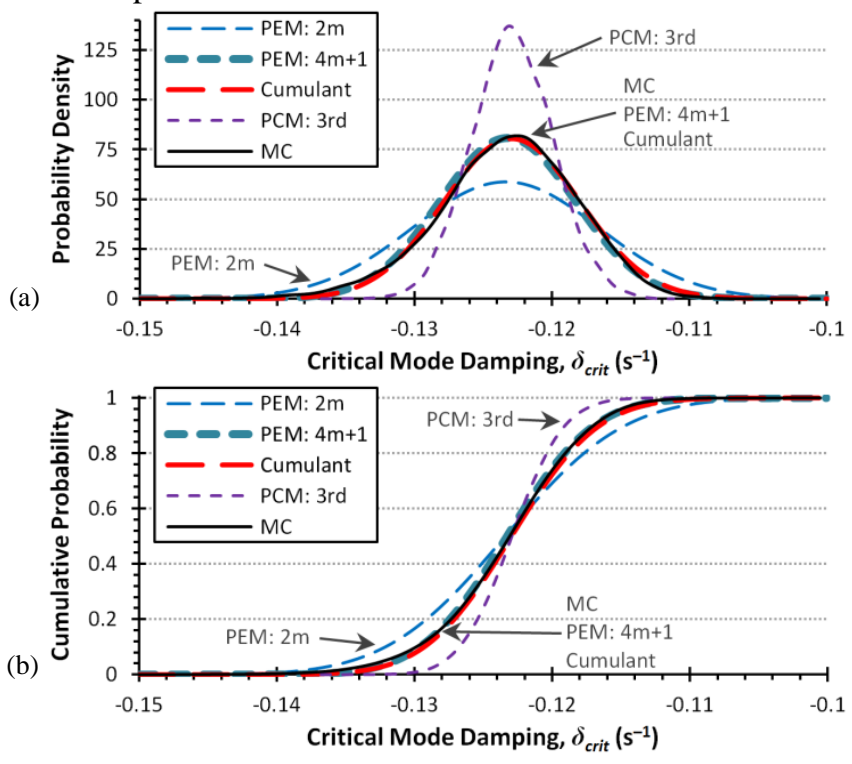

Fig. 3: (a) Pdfs, and (b) $c d f s$ of $\sigma_{\text {crit }}$ produced for $5 \%$ input uncertainty.

\section{4) Effect of Level of Uncertainty}

The values of $\varepsilon_{A R M S}$ are collated in Table 2, not only for the $5 \%$ variation shown in Fig. 3, but also for $2.5 \%$ and $7.5 \%$ input variability. These figures enable a thorough analysis of the suitability of different efficient estimation methods for probabilistic small-disturbance stability studies.

As suggested by Fig. 3, the $\varepsilon_{A R M S}$ values for the $4 m+1$ PEM variant and cumulant-based approach are similar (and low) for all levels of system uncertainty. It can also be seen that the distribution errors increase slightly with increasing uncertainty. The $2 \mathrm{~m}$ PEM variant displays the greatest sensitivity to the level of uncertainty. When input variability is $2.5 \%$, the $\varepsilon_{A R M S}$ value is acceptable at just $1.14 \%$. However, as 
the input variability rises to $5 \%$ and subsequently to $7.5 \%$, the distribution error values rise to $5.60 \%$ and $12.67 \%$ respective1y. No other efficient estimation method reduces so greatly in accuracy. All PCM models display approximately the same error $(7-8 \%)$ for all model orders at all levels of uncertain input variation. This efficient estimation approach appears to be largely unaffected by the level of uncertainty, however it is also typically the least accurate.

TABLE 2

VALUES OF $\varepsilon_{A R M S}$ FOR DifFERENT ESTIMATION METHODS WITH INCREASING LEVELS OF UNCERTAIN INPUT VARIABILITY

\begin{tabular}{|ll|ccc|}
\hline \multirow{2}{*}{ Method used } & \multicolumn{3}{|c|}{$\varepsilon_{\text {ARMS }}$} \\
& $2.5 \%$ & $5.0 \%$ & $7.5 \%$ \\
\hline \multirow{2}{*}{ PEM } & $2 m$ & $1.14 \%$ & $5.60 \%$ & $12.67 \%$ \\
& $4 m+1$ & $0.66 \%$ & $1.00 \%$ & $1.93 \%$ \\
\hline \multicolumn{2}{|c|}{ Cumulant } & $0.63 \%$ & $1.47 \%$ & $1.84 \%$ \\
\hline \multirow{2}{*}{ PCM } & $1^{\text {st }}$ Order & $7.80 \%$ & $7.71 \%$ & $8.25 \%$ \\
& $2^{\text {nd }}$ Order & $7.56 \%$ & $7.21 \%$ & $7.28 \%$ \\
& $3^{\text {rd }}$ Order & $7.38 \%$ & $7.59 \%$ & $7.19 \%$ \\
\hline
\end{tabular}

A common practical measure of distribution fit is the $90 \%$ confidence interval (the range between the $5^{\text {th }}$ and $95^{\text {th }}$ percentiles). Plotting these ranges for all efficient estimation techniques in Fig. 4, the best estimations produced by the cumulant and $4 m+1$ PEM approach are clearly evident.

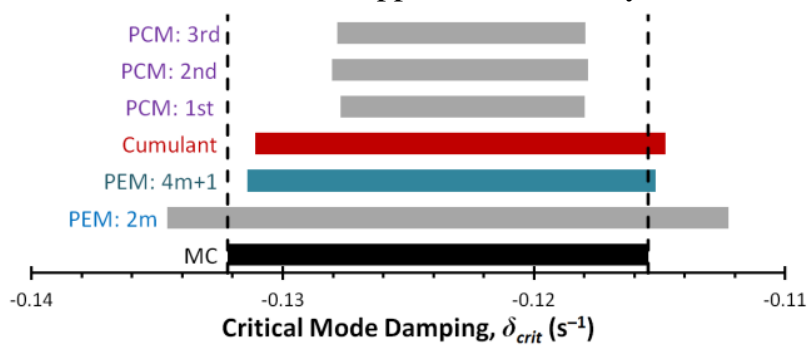

Fig. 4: $90 \%$ confidence intervals of $\delta_{\text {crit }}$ for $5 \%$ input uncertainty.

\section{Accuracy and Efficiency}

The results obtained have shown that the cumulant and $4 m+1$ PEM variant consistently provide the most accurate results. It is also important to assess this error against the efficiency of the methods when assessing which technique is most suitable for fast probabilistic small-disturbance stability studies.

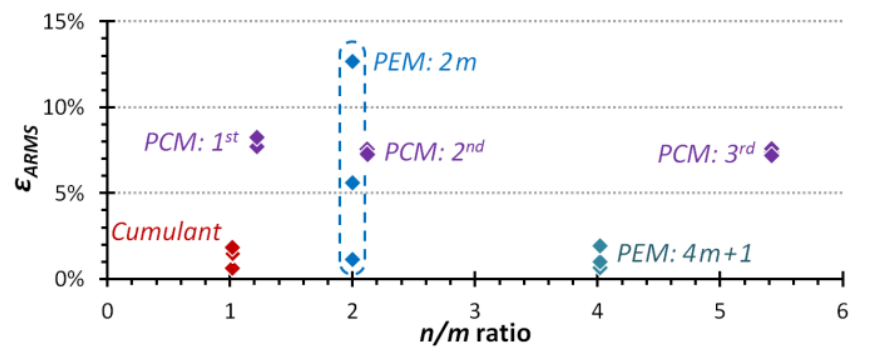

Fig. 5: Accuracy and efficiency of different techniques.

Fig. 5 shows the values of the distribution error (at all considered levels of uncertainty) plotted against the $n / m$ ratio, where $n$ is the number of full deterministic studies required to complete the analysis, and $m$ is the number of uncertainties.
Results in the bottom left of this plot display both low error and low computational requirements - the characteristics desired from such techniques. It is clear that although both the cumulant and $4 m+1$ PEM variant are comparable in terms of error performance, the cumulant approach is approximately four times more efficient. It should be noted that all methods demonstrate considerable efficiency savings over the traditional numerical MC approach. The least efficient method used here (the $3^{\text {rd }}$ order PCM model) still requires just $2.71 \%$ of the number of full deterministic studies required by the MC approach. For the most efficient method - the cumulant approach - this drops to $0.51 \%$.

\section{DiSCUSSION AND RECOMMENDATIONS}

Based on the results obtained within this work, it is recommended that the cumulant-based approach is used as an efficient estimation technique in future probabilistic smalldisturbance stability studies. This approach is the most efficient technique considered (with an $n / m$ ratio of approximately one) and it consistently produces results with low error.

It should be noted that the $4 m+1$ PEM variant also provides results with low error, albeit with lower efficiency than the cumulant approach. In [9], the $2 m+1$ PEM variant was shown to provide results as accurate as the $4 m+1$ variant, improving the efficiency of the method. This variant however, cannot be used with normally distributed uncertainties if $m>3$ [21] and it was, therefore, unsuitable for application in this study. Although the PEM approach does not impose a parametric distribution for the output, it was found that estimated moments above second order were too erroneous to include. The modeling of output distributions was therefore effectively restricted to Gaussian. If outputs are known to be non-Gaussian, then the cumulant approach should be used as it estimates high order moments more accurately.

It should also be noted that the cumulant method estimates the mean output value as the output when all inputs are at their mean values. For this test network this did not lead to any significant error, however in some systems it has been observed that this results in inaccuracies. In these cases, alternative methods may be required to maintain accuracy compromising on efficiency. The cumulant method is also dependent on the accuracy of the sensitivity term in (14) and may become less accurate if the system moves far from the linear region of behaviour. This could occur (though it was not observed during this study) during stressed conditions, such as very high loading, and requires further investigation. Finally, it was observed that all the considered techniques are able to reproduce the mean values (first raw moment) with very low error. If only mean values are required then any method would suffice - though the $2 \mathrm{~m}$ PEM variant should be avoided when input uncertainty variability increases.

\section{CONCLUSIONS}

The paper presented a comparative investigation of a number of efficient estimation techniques in order to assess their suitability for probabilistic small-disturbance stability studies of large uncertain power systems. The analysis presented here 
is not only the first comparison of different efficient estimation techniques for these type of studies but also the first analysis of the way in which the level of uncertainty affects the accuracy of the different proposed techniques. The accuracy of the methods has been assessed through comparisons with traditional numerical Monte Carlo simulation. Errors have been compared for both descriptive moments and also entire probability distributions of damping of critical electromechanical mode.

It has been established that the cumulant-based analytical approach (utilizing a numerical approximation to calculate system sensitivities) is the most suitable method for such studies. It provides the highest efficiency, best accuracy, and can be used with non-parametric distributions (both input and output). Furthermore, although not investigated within this work, it has been shown in the past that input correlation can also be included in the formulation of this method [11], [12]. The study enables more informed decisions to be made when selecting efficient estimation methods for probabilistic small disturbance stability studies of large uncertain power systems. These efficient techniques could also be used to gather further statistical information about the system modes (such as participation factors or residual values) that could enable probabilistic controller design.

\section{REFERENCES}

[1] S. M. Ustinov, J. V. Milanović, and V. A. Maslennikov, "Inherent dynamic properties of interconnected power systems," Int. J. Electr. Power Energy Syst., vol. 24, no. 5, pp. 371-378, Jun. 2002.

[2] G. Rogers, Power System Oscillations. Norwell: Kluwer Academic Publishers, 2000.

[3] J. L. Rueda, D. G. Colome, and I. Erlich, "Assessment and Enhancement of Small Signal Stability Considering Uncertainties," IEEE Trans. Power Syst., vol. 24, no. 1, pp. 198-207, 2009.

[4] R. Arrieta, M. A. Rios, and A. Torres, "Contingency Analysis and Risk Assessment of Small Signal Instability," Power Tech, 2007 IEEE Lausanne. pp. 1741-1746, 2007.

[5] C. K. Pans, Z. Y. Dong, P. Zhang, and X. Yin, "Probabilistic analysis of power system small signal stability region," in International Conference on Control and Automation. ICCA '05., 2005, vol. 1, pp. 503-509.

[6] R. C. Burchett and G. T. Heydt, "Probabilistic Methods For Power System Dynamic Stability Studies," IEEE Trans. Power Appar. Syst., vol. PAS-97, no. 3, pp. 695-702, May 1978.

[7] G. Verbic and C. A. Canizares, "Probabilistic Optimal Power Flow in Electricity Markets Based on a Two-Point Estimate Method," IEEE Trans. Power Syst., vol. 21, no. 4, pp. 1883-1893, 2006.

[8] C.-L. Su, "Probabilistic load-flow computation using point estimate method," IEEE Trans. Power Syst., vol. 20, no. 4, pp. 1843-1851, 2005.

[9] J. M. Morales and J. Perez-Ruiz, "Point Estimate Schemes to Solve the Probabilistic Power Flow," IEEE Trans. Power Syst., vol. 22, no. 4, pp. 1594-1601, Nov. 2007.

[10] J. Rueda and I. Erlich, "Probabilistic framework for risk analysis of power system small-signal stability," Proc. Inst. Mech. Eng. Part O J. Risk Reliab., vol. 226, no. 1, pp. 118-133, 2012.

[11] S. Q. Bu, W. Du, H. F. Wang, Z. Chen, L. Y. Xiao, and H. F. Li, "Probabilistic Analysis of Small-Signal Stability of Large-Scale Power Systems as Affected by Penetration of Wind Generation," IEEE Trans. Power Syst., vol. 27, no. 2, pp. 762-770, 2012.

[12] K. W. Wang, C. Y. Chung, C. T. Tse, and K. M. Tsang, "Improved probabilistic method for power system dynamic stability studies," IEE Proc. Gener. Transm. Distrib., vol. 147, no. 1, pp. 37-43, 2000.

[13] P. Zhang and S. T. Lee, "Probabilistic load flow computation using the method of combined cumulants and Gram-Charlier expansion," IEEE Trans. Power Syst., vol. 19, no. 1, pp. 676-682, 2004.

[14] A. Schellenberg, W. Rosehart, and J. Aguado, "Cumulant-based probabilistic optimal power flow (P-OPF) with Gaussian and gamma distributions," IEEE Trans. Power Syst., vol. 20, no. 2, pp. 773-781, 2005.

[15] M. A. Tatang, "Direct incorporation of uncertainty in chemical and environmental systems," Massachusetts Institute of Technology, 1995.

[16] J. R. Hockenberry and B. C. Lesieutre, "Evaluation of Uncertainty in Dynamic Simulations of Power System Models: The Probabilistic Collocation Method," IEEE Trans. Power Syst., vol. 19, no. 3, pp. 1483-1491, Aug. 2004.

[17] R. Preece, N. C. Woolley, and J. V. Milanovic, "The Probabilistic Collocation Method for Power System Damping and Voltage Collapse Studies in the Presence of Uncertainties," IEEE Trans. Power Syst., vol 28, no. 3, pp. 2253-2262, 2013.

[18] P. Kundur, Power System Stability and Control. New York: McGrawHill, Inc., 1994.

[19] A. Papoulis, Probability, Random Variables and Stochastic Processes, Second. New York: McGraw Hill, 1984, p. 575.

[20] R. Y. Rubinstein, Simulation and the Monte Carlo Method. New York: Wiley, 1981.

[21] H. P. Hong, "An efficient point estimate method for probabilistic analysis," Reliab. Eng. Syst. Saf., vol. 59, no. 3, pp. 261-267, Mar. 1998.

[22] K. S. Li, "Point-Estimate Method for Calculating Statistical Moments," J. Eng. Mech., vol. 118, no. 7, pp. 1506-1511, Jul. 1992.

[23] M. E. Harr, "Probabilistic estimates for multivariate analyses," Appl. Math. Model., vol. 13, no. 5, pp. 313-318, May 1989.

[24] J. M. Morales, L. Baringo, A. J. Conejo, and R. Mínguez, "Probabilistic power flow with correlated wind sources," IET Gener. Transm. Distrib. vol. 4, no. 5, p. 641, 2010.

[25] H. Cramér, Mathematical Methods of Statistics. Princeton: Princeton University Press, 1957

[26] P. Beckmann, Orthogonal Polynomials for Engineers and Physicists. Boulder, CO: Golem Press, 1973

[27] G. W. Gautschi, "Algorithm 726: ORTHPOL - A package of routines for generating orthogonal polynomials and Gauss-type quadrature rules," ACM Trans. Math. Softw., vol. 20, pp. 21-62, 1994.

[28] P. Davis and P. Rabinowitz, Methods of Numerical Integration, Second Ed. Mineola: Dover Publications, 2007.

[29] B. Pal and B. Chaudhuri, Robust Control in Power Systems. New York: Springer Inc., 2005

[30] R. D. Zimmerman, C. E. Murillo-Sanchez, and R. J. Thomas, "MATPOWER: Steady-State Operations, Planning, and Analysis Tools for Power Systems Research and Education," IEEE Trans. Power Syst., vol. 26, no. 1, pp. 12-19, Feb. 2011.

[31] R. Preece, J. V. Milanovic, A. M. Almutairi, and O. Marjanovic, "Damping of Inter-Area Oscillations in Mixed AC/DC Networks Using WAMS Based Supplementary Controller," IEEE Trans. Power Syst., pp. 1-1, 2012.

[32] A. W. Bowman and A. Azzalini, Applied Smoothing Techniques for Data Analysis. New York: Oxford University Press, 1997.

[33] D. Maillard, "A User's guide to the Cornish Fisher Expansion." Social Science Research Network, 2012.

Robin Preece (GS'10, M'13) received his BEng degree in Electrical and Electronic Engineering in 2009 and his $\mathrm{PhD}$ degree in 2013, both from the University of Manchester, UK. He is currently working as a Research Associate at the same institution investigating risk and uncertainty with respect to the stability of future power systems.

Kaijia Huang received BEng degree in Electrical and Electrical Engineering from both North China Electrical Power University and Cardiff University, UK, in 2012. He is currently working towards the degree of MSc in Electrical Power Systems Engineering at the University of Manchester, UK.

Jovica V. Milanović (M'95, SM'98, F'10) received his Dipl.Ing and his MSc degrees from the University of Belgrade, Yugoslavia, his $\mathrm{PhD}$ degree from the University of Newcastle, Australia, and his Higher Doctorate (DSc degree) from The University of Manchester, UK, all in Electrical Engineering. Currently, he is a Professor of electrical power engineering and Deputy Head of the School of Electrical and Electronic Engineering at The University of Manchester, UK, Visiting Professor at the University of Novi Sad, Novi Sad, Serbia and Conjoint Professor at the University of Newcastle, Newcastle, Australia. 